\title{
Coexisting overwintering strategies in Daphnia pulex: Clonal differences in sexual reproduction
}

\author{
Winfried Lampert ${ }^{1,2, *}$, Kathrin P. Lampert ${ }^{3}$ and Petter Larsson ${ }^{1}$
}

With 6 figures and 4 tables

\begin{abstract}
Using two sets of clones of Daphnia pulex derived from overwintering parthenogenetic females and neonates hatched from dormant embryos, we tested for a genetic basis of differing overwintering strategies. While in an earlier study we had tested if the two clonal groups differed with respect to their growth rates, which would give them different opportunities during the spring development, we now performed experiments on possible differences in traits related to sexual reproduction (i.e., male production and ephippia formation). Our working hypothesis was that clones isolated from the sediment egg bank show a greater tendency to reproduce sexually than females overwintering in the open water. Although individual clones showed large differences in the proportion of males among offspring under short-day conditions (zero to $>40 \%$ ), the clonal groups did not differ significantly. Ephippia production was also significantly different among clones, but due to the large variances the group means did not differ. Ephippia production and male production in individual clones were not correlated, hence the tendencies to produce males or ephippia varied independently. Although there were considerable differences in the mean reproductive characteristics between the groups, the low power of the tests prevented support of any of the hypotheses. Rather, it seems that there is incomplete temporal differentiation while some females may follow a mixed strategy implying the production of sexual eggs as insurance against catastrophic events and successive attempts to survive the winter as parthenogenetic adults, which would result in a reproductive advantage during early spring.
\end{abstract}

Key words: clones, dual reproduction mode, pathenogenetic females, dormant embryos, ephippia production, fertilization efficiency.

\section{Introduction}

In permanent water bodies, Daphnia, due to their dual reproduction mode, have two principal options to survive the winter: (1) They can overwinter as parthenogenetic females in the open water. This strategy bears the risk of not surviving the harsh winter conditions or catastrophic events like oxygen depletion under ice. However, if a female survives it has the advantage of immediate reaction to improving temperature and food conditions in early spring, which results in a large number of early offspring. (2) Being cyclic par- thenogens, Daphnia can also overwinter as dormant embryos encased in an ephippium as a result of sexual reproduction. This strategy can be seen as bet-hedging, insuring survival during catastrophic event. The disadvantage is slower population growth in spring as the new founder generation must first hatch in response to an external stimulus and then grow to adulthood. The prevalence of the two strategies in a particular water body may depend on the predictability of abiotic and biotic environmental factors. Examples are discussed in Lampert et al. (2010).

\footnotetext{
Authors' addresses:

${ }^{1}$ University of Bergen, Department of Biology, Bergen, Norway.

${ }^{2}$ Present address: Max Planck Institute for Evolutionary Biology, Postfach 165, 24302 Plön, Germany.

${ }^{3}$ University of Bochum, Evolutionary Ecology and Biodiversity of Animals, Bochum, Germany.

*Corresponding author; lampert@evolbio.mpg.de
} 
In the winter 2006/07 we found both strategies to coexist in a population of Daphnia pulex in Myravann, a small, mesotrophic Norwegian lake with negligible fish planktivory. The absence of pelagic fish (Knudsen et al. 2006) made Myravann a rather unique system, where the life history of a large Daphnia species must have been shaped by changing environmental conditions rather than by fish predation. To experimentally study the question of a genetic basis for the overwintering strategy, we established two groups of clones, one from females overwintering in the open water, and a second one from dormant embryos isolated from ephippia collected in the field and hatched in the laboratory (Lampert et al. 2010). The hypothesis was that some clones in the lake are selected for longterm parthenogenetic existence, while other clones are selected for an annual cycle with sexual reproduction in fall and overwintering in ephippia. The alternative hypothesis was that clones are not selected for specific overwintering strategies. Instead each clone alternates between parthenogenetic and sexual reproduction, contributes to the dormant egg bank, and nevertheless attempts to overwinter in the open water.

Neutral molecular markers (microsatellites) discriminated the individual clones but did not detect higher genetic relatedness within the groups than between the groups. Also, despite large differences between clones, group means of traits related to the performance in spring (growth reaction norms to increasing food concentrations and maximum juvenile growth rates) did not differ significantly. There was no correlation between the genetic relatedness of individual clones and growth rates (Lampert et al. 2010).

However, this does not exclude the possibility that the production of dormant stages is a quantitative process, i.e. the tendency for sexual reproduction varies between clones. The "pure" strategies may just be the extremes of a distribution, and the mean differences for the traits measured were eventually too small to be detected in samples of 10 clones for each group. Hence we decided to run additional experiments and measure the tendency to reproduce sexually for the individual clones directly. These experiments tested the hypothesis that the clones originating from dormant stages had on average a higher tendency to reproduce sexually than the clones originating from overwintering females.

The successful production of dormant embryos requires two steps (Zaffagnini 1987). First, some females need to be environmentally stimulated to produce male offspring instead of females. Second, females must be stimulated to produce two sexual ("amphigonic") eggs that undergo meiosis and can be fertilized in the ovary by the males. The timing of the two steps is important. The induction of males must happen about two weeks before the induction of sexual eggs as the males must mature in order to fertilize them (Larsson 1991). Consequently, although the same environmental factors have been demonstrated to induce both processes of sexual reproduction, the stimuli for the production of males and meiotic eggs cannot be perfectly identical, which makes laboratory experiments complicated. While male production first of all seems to be induced by a short photoperiod (Hobaek \& Larsson 1990), reduced food conditions and crowding may be required for ephippia formation (Kleiven et al. 1992, La Montagne \& McCauley 2001). Hence we used two experimental approaches. One was a population approach studying the clonal variation in the induction of ephippia during periods with changing environmental conditions that affect both the production of sexual eggs and males. The second was an individual approach to measure clonal differences in the production of males at constant conditions.

\section{Material and methods}

\section{General}

This study is based on the comparison of two groups of clones of Daphnia pulex isolated from Myravann, a small mesotrophic, stratifying lake lacking planktivorous fish in the outskirts of Bergen, Norway. The lake is small (61.5 ha), with a mean depth of $7.6 \mathrm{~m}$ and a maximum depth of $18 \mathrm{~m}$. Although it freezes regularly in winter, the ice cover is variable. When we sampled the experimental clones, Daphnia pulex was the dominant cladoceran species, accompanied by D. longispina. Chaoborus flavicans larvae were abundant. One group (W) consisted of 11 clones isolated from the open water in December 2006 and was considered to be overwintering as adult females. The second group (E) had 10 clones isolated from hatched ephippia that had been collected from the lake sediments (6-7 m depth) in early March 2007, shortly after ice break. This group had been overwintering as sexual, dormant embryos. The clones were propagated parthenogenetically under continuous light in the laboratory for the successive three years at approximately $20^{\circ} \mathrm{C}$.

We used membrane-filtered, aged water from Myravann for the cultures as well as for the experiments. Daphnia were fed green algae (Scenedesmus obliquus) grown in chemostats. Detailed information on the lake, the isolation of clones and their genetic relationship, and on the cultivation of Daphnia and food algae can be found in Lampert et al. (2010).

\section{Experiment 1}

This experiment aimed to find differences in the production of ephippia between the clonal groups. As the induction of sexual reproduction is a complex process that includes various stimuli 


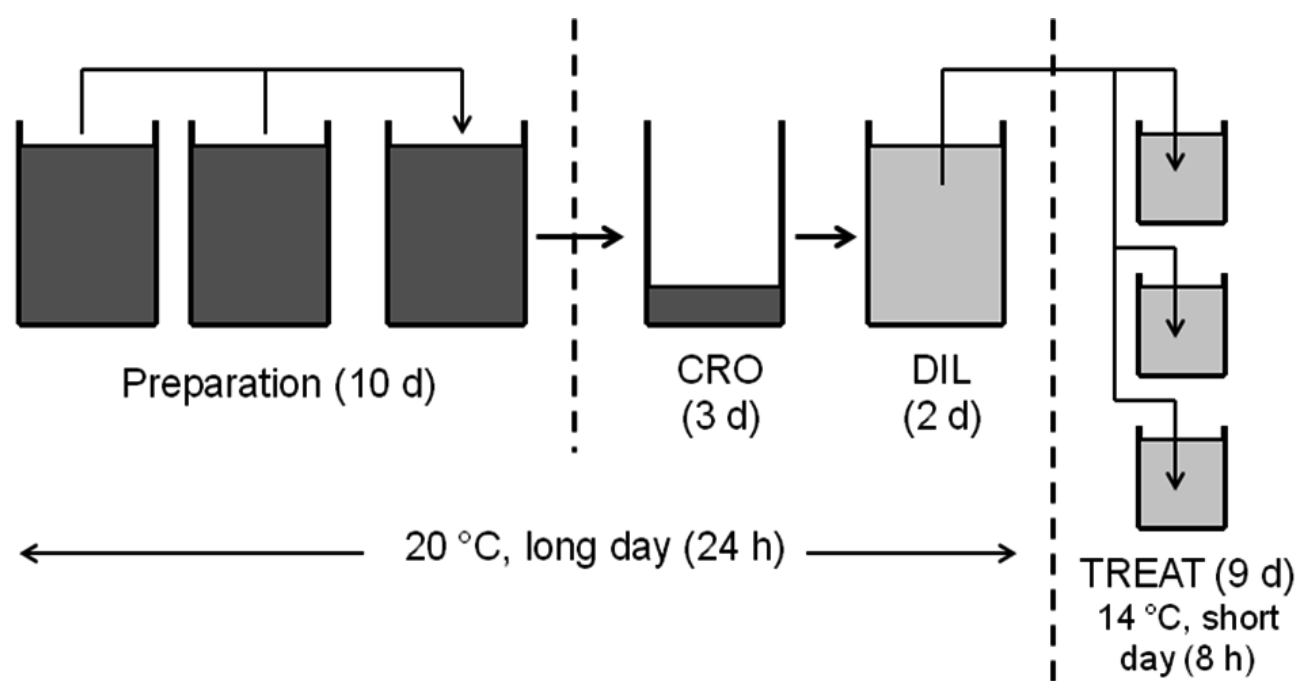

Fig. 1. Schematic representation of Expt. 1. Large vessels represent large (1 L) jars, small vessels contain 300 mL. Shading symbolizes high (dark) or low (light) food concentration. Phases: $\mathrm{CRO}=$ crowding; DIL $=$ dilution; TREAT $=$ treatment.

we ran an experimental protocol executing a sequence of environmental changes (crowding - food reduction - short days and low temperature; Fig. 1). After a preparatory phase of 10 days, we combined approximately 100 individuals of each D. pulex clone from all size classes into separate 1 litre glass jars (one jar per clone) at a food concentration of $2 \mathrm{mg}$ carbon $\mathrm{L}^{-1}$. As crowding is considered a factor inducing both male and ephippia production in Daphnia (Larsson 1991), we started with a crowding treatment. We siphoned out $75 \%$ of the water from each jar through a $100 \mu \mathrm{m}$ mesh net to concentrate the Daphnia fourfold. For the next 3 days Daphnia were kept under crowding conditions, but they were fed three times a day to maintain sufficient food conditions. This period was followed by refilling the jars with membrane-filtered water and no feeding, which resulted in a sudden drop of the food concentration. A sudden decrease in food availability is considered to stimulate ephippia production (La Montagne \& McCauley 2001). This "dilution" period lasted 2 days. So far, the jars had been kept at $20^{\circ} \mathrm{C}$ under continuous dim light. Daphnia populations grew during this period but population size and composition could not be assessed quantitatively. However, all ephippia shed during the 5 days of the crowding and dilution phase were collected. The majority of ephippia collected in the crowding treatment had probably been induced already during the preparatory phase. Many of the ephippia were empty indicating that the sexual eggs had not been fertilized. We did not discriminate between empty and full ephippia as we took the presence of an ephippium as the indication of a female's readiness for sexual reproduction regardless of the presence of males.

Two days in the dilute food conditions without additional feeding were followed by a period of 9 days under drastically changed conditions further on called "treatment" phase (Fig. 1). The contents of the large jars were split and distributed to three $300 \mathrm{~mL}$ jars each. These jars were then exposed to reduced temperature $\left(14^{\circ} \mathrm{C}\right)$ and short days $(8: 16 \mathrm{~h} \mathrm{~L}: \mathrm{D})$ in a temperature controlled cabinet. Each jar was fed a total of $0.35 \mathrm{mg} \mathrm{C}$ Scenedesmus twice a day at the beginning and at the end of the light phase, which resulted in limiting food conditions but not in severe starvation that would prevent reproduction. At the end of the experiment, all Daphnia and ephippia were recovered and preserved in sugar formalin (Haney \& Hall 1971) for later processing. All samples were counted completely under a dissecting microscope. As the size at maturation of the clones varied in the laboratory experiments between 1.9 and $2.1 \mathrm{~mm}$, we discriminated juvenile (body length $<2 \mathrm{~mm}$ ) and adult females, males and ephippia (shed or still attached to the female), but we did not consider decayed carcasses. To compensate for varying population densities, we calculated the number of ephippia per adult produced during the 9 days and the proportion of males in the total population for each jar.

The two phases of the experiment (crowding/dilution and treatment) resulted in different data sets. During the crowding/ dilution phase we only collected shed ephippia from single populations per clone. At the end of the treatment phase we had three replicate populations per clone, and we were able to count all females, males, and ephippia present. The sum of all ephippia found in both phases resulted in a single figure of ephippia production per clone.

\section{Experiment 2}

As the production of males was rather variable and is supposed to be clone specific (Larsson 1991), we performed a second experiment aiming to estimate the tendency of male production in the different clones.

Some large egg-carrying females of each clone were isolated in $300 \mathrm{~mL}$ jars, and neonates were collected as soon as they had been released. For each clone, 5 neonates each were combined in six $50 \mathrm{~mL}$ glass jars containing food suspension of $1 \mathrm{mg} \mathrm{C} \mathrm{L}{ }^{-1}$. They were kept at $20^{\circ} \mathrm{C}$ under short-day $(8 \mathrm{~h}$ light) conditions, which is supposed to be a strong stimulus for male production (Hobaek \& Larsson 1990), and transferred to fresh containers every day. After four days, when the females were grown up, the numbers were reduced to 3 per jar. Females were transferred to new medium daily until they released the first neonates. For the successive 4 days they were transferred every second day. This resulted in three samples of neonates from each jar. After adult females had been transferred, neo- 
nates were kept in the jars for 5 days to let them grow and make sex determination more reliable. They were then preserved and sexed later under a dissecting microscope. To compensate for the loss of individual adults during the experiment we pooled offspring from the three jars and the three sampling dates and calculated the mean proportion of males in the neonate population.

\section{Statistics}

Parametric statistical analyses were performed using the NCSS package (Hines 2000). To estimate the effects of group (E and $\mathrm{W}$ ) and clone (nested in group) on population and reproductive characteristics at the end of the "treatment" phase of Expt.1, we used two-way ANOVA after testing for normality and equal variances. The three jars per clone were considered replicates as they were random subsamples from the same population, and the treatment phase implied a separate period of drastically changed conditions. NCSS also reported the power of the tests.

The three jars were pooled when group means were calculated so that $n$ reflects always the number of clones. As the distributions of reproductive characteristics within the groups was eventually skewed, and contained a considerable proportion of zero values, we estimated standard deviations using a bootstrap method (Howell 2000) with 10.000 draws. Group means were compared by a $t$-test based on a randomization test (Howell 2000 ) with 10.000 runs. This programme reported also the effect size that could be used to run a power analysis $\left(\mathrm{G}^{*}\right.$ Power, v. 3.1.3; Faul et al. 2007).

\section{Results}

\section{Experiment 1}

Although genetic studies (Lampert et al. 2010) had shown that two pairs of the $11 \mathrm{~W}$ clones had to be considered identical on the basis of neutral markers, it turned out that these pairs differed with respect to reproductive characteristics (Table 1). The paired clones differed in the number of ephippia produced (W14 did not produce any ephippia) as well as in the production of males. Therefore, we decided to include all clones in the analysis, which reflects the full population sampled.

Table 1. Reproductive characteristics of the two pairs of clones that were considered "identical" as they could not be genetically discriminated by 12 polymorphic microsatellite loci (Lampert et al. 2010).

\begin{tabular}{lccccc}
\hline & \multicolumn{2}{c}{ Pair 1 } & & \multicolumn{2}{c}{ Pair 2 } \\
\cline { 2 - 3 } \cline { 6 - 6 } Clones & W1 & W5 & & W4 & W14 \\
\hline Experiment 1 & & & & & \\
Total \# of ephippia & 9 & 71 & & 28 & 0 \\
\# of ephippia in TREAT & 9 & 15 & & 6 & 0 \\
Ephippia/Adult & 0.105 & 0.170 & & 0.048 & 0 \\
Males (\%) & 0.09 & 0 & & 0.14 & 0.24 \\
\hline Experiment 2 & & & & \\
Males (\% offspring) & 25.3 & 14.9 & & 28.6 & 0 \\
\hline
\end{tabular}

The total number of ephippia collected during the crowding, dilution, and treatment phase varied considerably between clones. In both clonal groups (E and W) two clones did not produce any ephippia while the maximum per clone was in the order of 80 (Fig. 2). Nearly all clones that produced low ephippia numbers $(<10$ in total) did so either in the dilution/crowding or in the treatment phase alone. On average, the proportions of total ephippia produced during the dilution/ crowding phase were $73.3 \%$ and $68.4 \%$ for the E and $\mathrm{W}$ clones, respectively, although the distributions within groups differed (Fig. 2). The average ( \pm 1 SD) number of total ephippia per clone was $20.5 \pm 26.2$ in the $\mathrm{E}$ clones and $12.6 \pm 20.1$ in the $\mathrm{W}$ clones (Table 2).

The mean population characteristics of the two groups at the end of the treatment phase have been compiled in Table 2. Mean population densities (per jar) at the end of the experiment were remarkably similar. The density of approximately 1000 individuals per litre was very high. However, both groups comprised about $87 \%$ juveniles indicating that the populations had been growing. In contrast, the mean proportions of males were very low for both groups and the standard variations were high ( $>180 \%$ of the mean) due to the large clonal variation. Each of the two groups contained clones without any males. Only two E clones and one $\mathrm{W}$ clone reached male proportions above $1 \%$ (Fig. 3).

Two-way ANOVAs with "group" as fixed factor and "clone" nested in "group" for the final parameters population density, proportion of juveniles, proportion of males, and ephippia per adult (E/A) showed no significant differences between groups (Table 3), although there were significant differences between the individual clones (except for juveniles). However, the probability of a type II error for the "group" factor was relatively high as the power (1- $\beta$ ) was low.

Table 2. Means ( \pm 1 SD) of population characteristics and reproductive characteristics for groups $E(n=10)$ and $W(n=11)$ in both experiments. SD estimated by bootstrapping.

\begin{tabular}{lcc}
\hline Group & $\mathrm{E}$ & $\mathrm{W}$ \\
\hline Experiment 1 & & \\
Total number of ephippia & $20.5(26.6)$ & $12.6(20.1)$ \\
Population density (ind. jar ${ }^{-1}$ ) & $351.7(71.2)$ & $350.8(50.2)$ \\
Juveniles (\%) & $86.6(4.4)$ & $87.1(3.0)$ \\
Males (\%) & $1.08(1.88)$ & $0.185(0.275)$ \\
Ephippia/Adult & $0.083(0.164)$ & $0.037(0.053)$ \\
\hline Experiment 2 & & \\
Male offspring (\%) & $13.5(16.3)$ & $20.7(12.1)$ \\
\hline
\end{tabular}



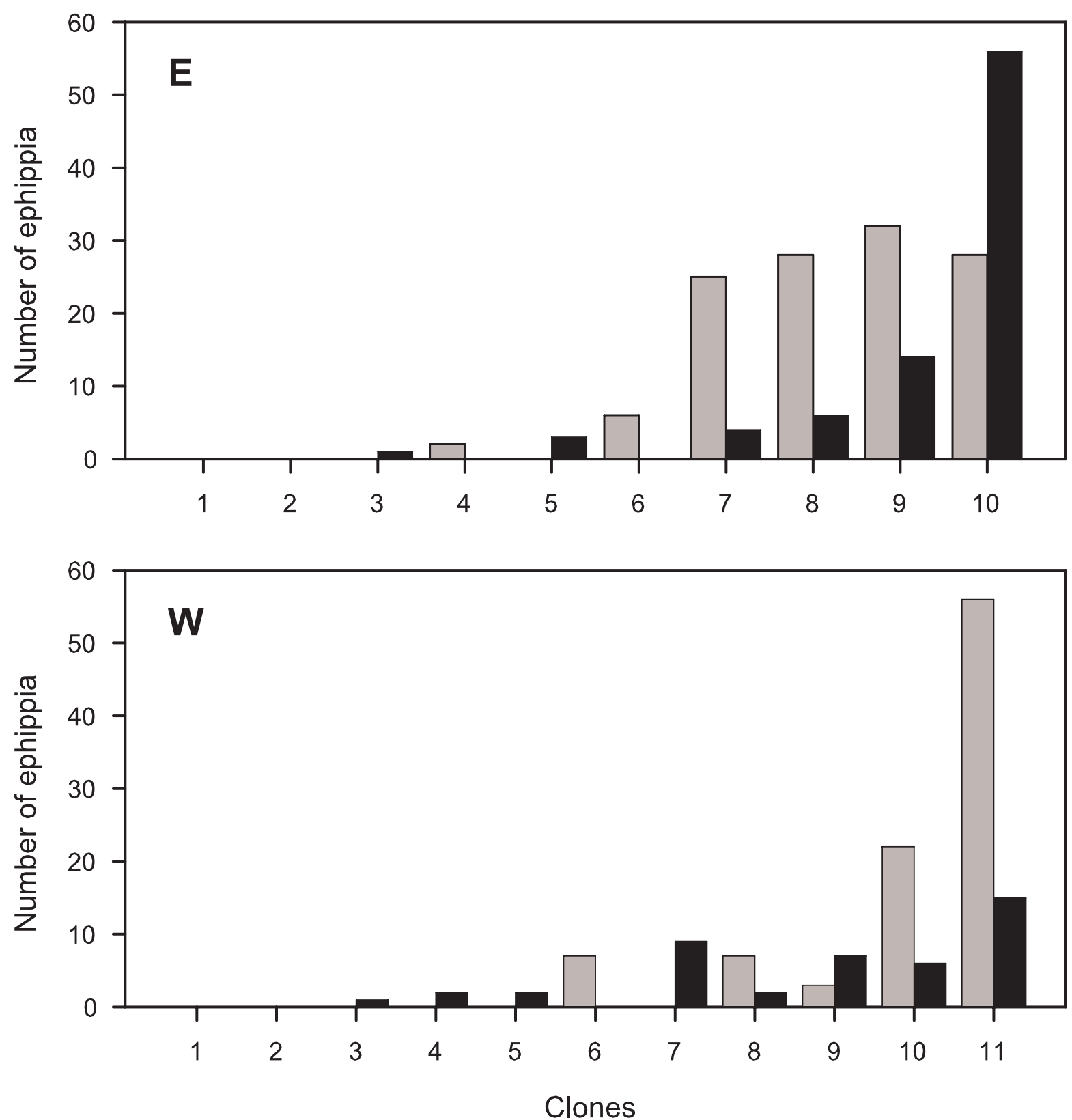

Fig. 2. Clonal variation of ephippia production in the two groups (E and $\mathrm{W}$ ) during the crowding/dilution phase (shaded bars) and during the treatment phase (black bars) of Expt.1. The clones are sorted within the groups according to increasing total ephippia numbers.

The great differences in ephippia production among clones are clearly visible in Fig. 2. It can also be seen that among the six clones that produced a total of more than 20 ephippia five produced the majority during the 5 days of the crowding/dilution phase. Nevertheless, there is a positive trend (Fig. 4) relating the total number of ephippia to the number of ephippia per adult at the end of the experiment (E/A) . However, clonal differences in male production were not related to E/A (Fig. 3). In particular, the clones with the highest $\mathrm{E} / \mathrm{A}$ ratios in the two groups produced only a single male $(<0.1 \%)$ in $\mathrm{E}$ and zero males in $\mathrm{W}$.

\section{Experiment 2}

Under short-day conditions, individual clones showed great variability in the production of males. The proportion of male offspring varied between 0 and $>40 \%$ 
Table 3. Two-way ANOVA testing for the effect of group ( $\mathrm{E}$ and $\mathrm{W}$ ) and clone (nested in group) on population and reproductive characteristics in Expt. 1. Asterisks indicate significant results at $\alpha=0.05$. MS $=$ mean square.

\begin{tabular}{|c|c|c|c|c|c|c|}
\hline Parameter & Effect & $\mathrm{df}$ & MS & $F$ & $p$ & power $(1-\beta)$ \\
\hline \multicolumn{7}{|c|}{ Total population } \\
\hline & Group & 1 & 11.4 & 0.00 & 0.978 & 0.050 \\
\hline & Clone (group) & 9 & 13886 & 3.36 & $0.003 *$ & \\
\hline & Error & 52 & 4130 & & & \\
\hline \multicolumn{7}{|c|}{ Juveniles (\%) } \\
\hline & Group & 1 & 3.9 & 0.23 & 0.646 & 0.071 \\
\hline & Clone (group) & 9 & 17.4 & 0.87 & 0.555 & \\
\hline & Error & 52 & 20.0 & & & \\
\hline \multicolumn{7}{|c|}{ Ephippia/Adult } \\
\hline & Group & 1 & 0.033 & 0.83 & 0.386 & 0.129 \\
\hline & Clone (group) & 9 & 0.039 & 3.14 & $0.004 *$ & \\
\hline & Error & 52 & 0.015 & & & \\
\hline \multicolumn{7}{|l|}{ Males (\%) } \\
\hline & Group & 1 & 12.5 & 2.76 & 0.131 & 0.318 \\
\hline & Clone (group) & 9 & 4.5 & 2.98 & $0.006^{*}$ & \\
\hline & Error & 52 & 1.5 & & & \\
\hline
\end{tabular}

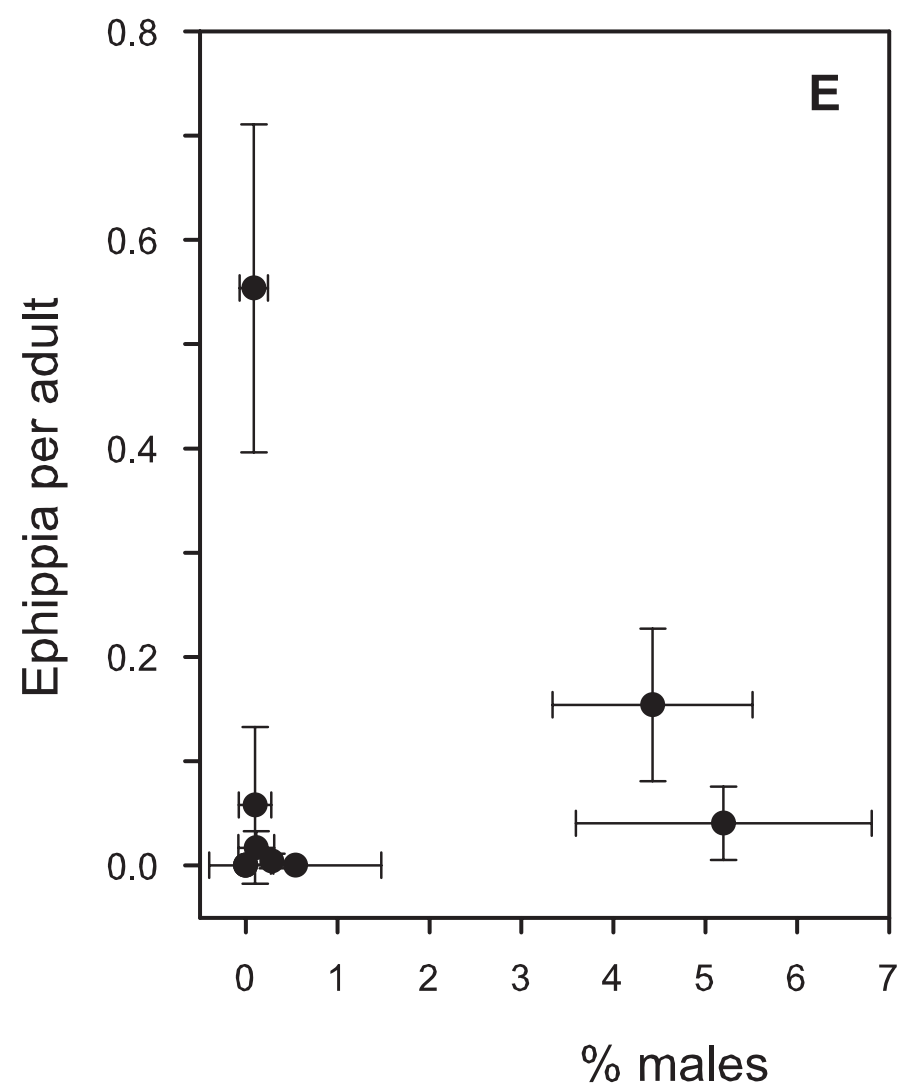

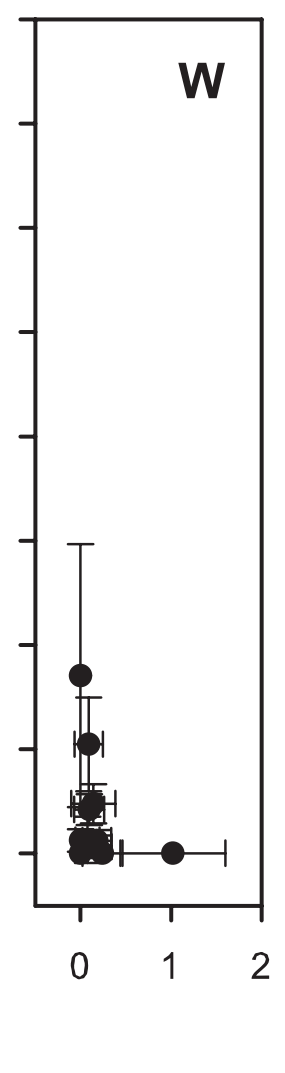

Fig. 3. Relationship between the proportion of males and the relative number of ephippia (E/A) found at the termination of Expt. 1 in the two clonal groups. Each dot represents the mean of one clone $\pm 1 \mathrm{SD}(\mathrm{n}=3)$.
(Fig. 5). The distribution differed as there were more E clones producing no males at all. Mean $( \pm$ SD) percentages in the controls were $20.7 \pm 12.1$ for the $\mathrm{W}$ clones and 13.5 \pm 16.3 for the E clones (Table 2).

\section{Comparison of group means}

There was no significant difference between group means for any of the characteristics listed in Table 2 (Table 4). For the population characteristics (popula- 
Fig. 4. Relationship between the total numbers of ephippia produced by different clones in Expt. 1 and the relative numbers $(\mathrm{E} / \mathrm{A})$ found at the termination of the treatment phase (mean $\pm 1 \mathrm{SD})$ in the two groups.

Fig. 5. Proportions of male neonates produced by individual clones in Expt. 2. Clones sorted according to increasing proportions within the two groups.
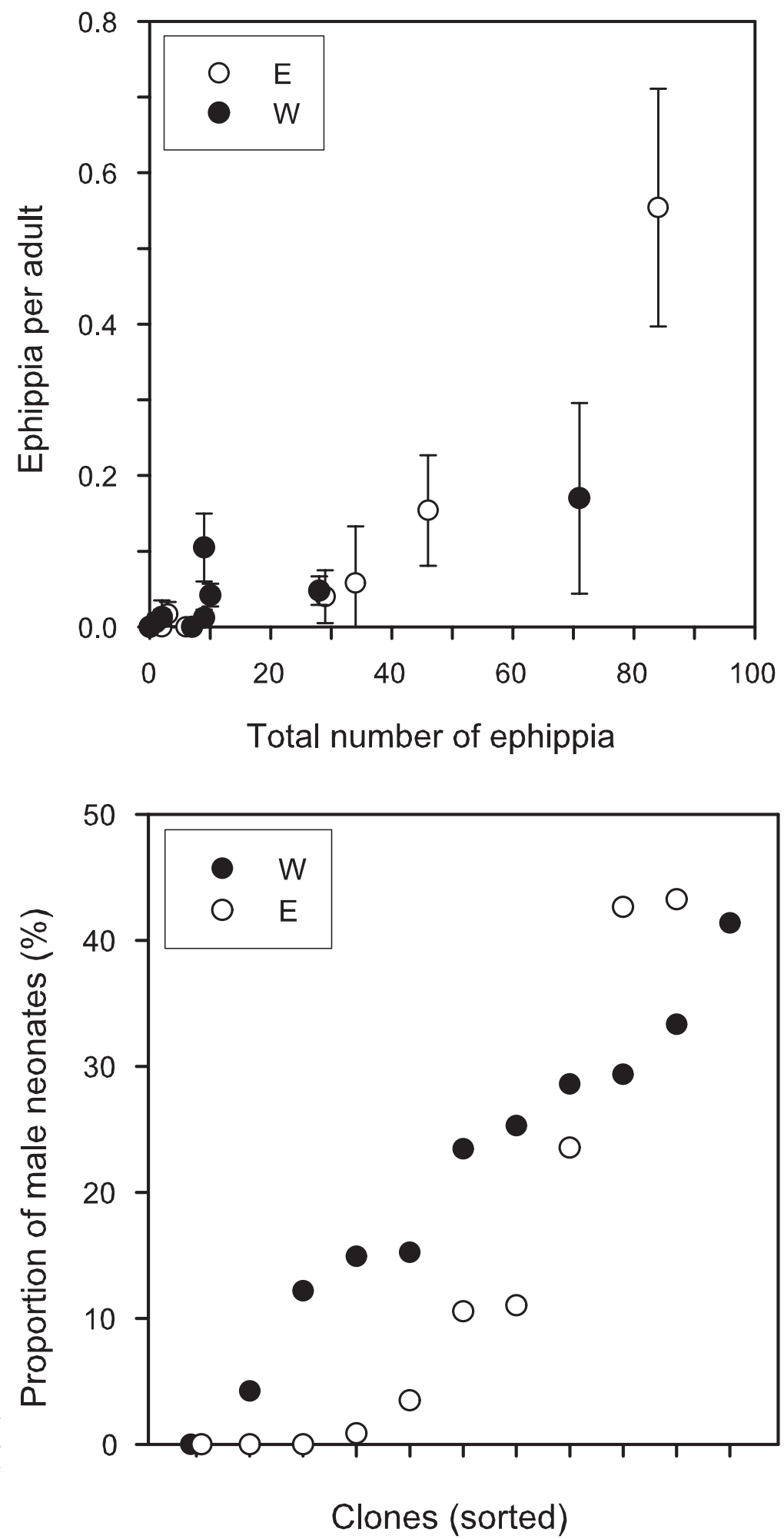

tion density, \% juveniles), this indicates that the experimental conditions for the two groups were identical, i.e. population growth rates did not differ between groups. For the reproductive characteristics we were not able to detect differences between group means, i.e. we had to accept the null hypothesis for male production and ephippia production despite relatively large differences between the means. However, a post hoc power analysis (Table 4) revealed that the power of the tests was low due to the very large within group 
Table 4. Statistics of the comparison of the group means (E: $n=10 ; \mathrm{W}: \mathrm{n}=11)$ of all measured characteristics in both experiments by a randomization method, t-test, and power analysis $(\alpha=0.05)$.

\begin{tabular}{lcccc}
\hline Parameter & $t$ & $p$ & effect size & power (1- $\beta$ ) \\
\hline Experiment 1 & & & & \\
Population density (ind. jar ${ }^{-1}$ ) & 0.030 & 0.978 & 0.007 & 0.050 \\
Juveniles (\%) & 0.289 & 0.783 & 0.063 & 0.052 \\
Males (\%) & 1.476 & 0.223 & 0.322 & 0.108 \\
Ephippa/Adult & 0.835 & 0.561 & 0.182 & 0.068 \\
Total ephippia & 0.735 & 0.477 & 0.160 & 0.064 \\
\hline Experiment 2 & & & & \\
Males (\%) & -1.100 & 0.280 & 0.240 & 0.082 \\
\hline
\end{tabular}
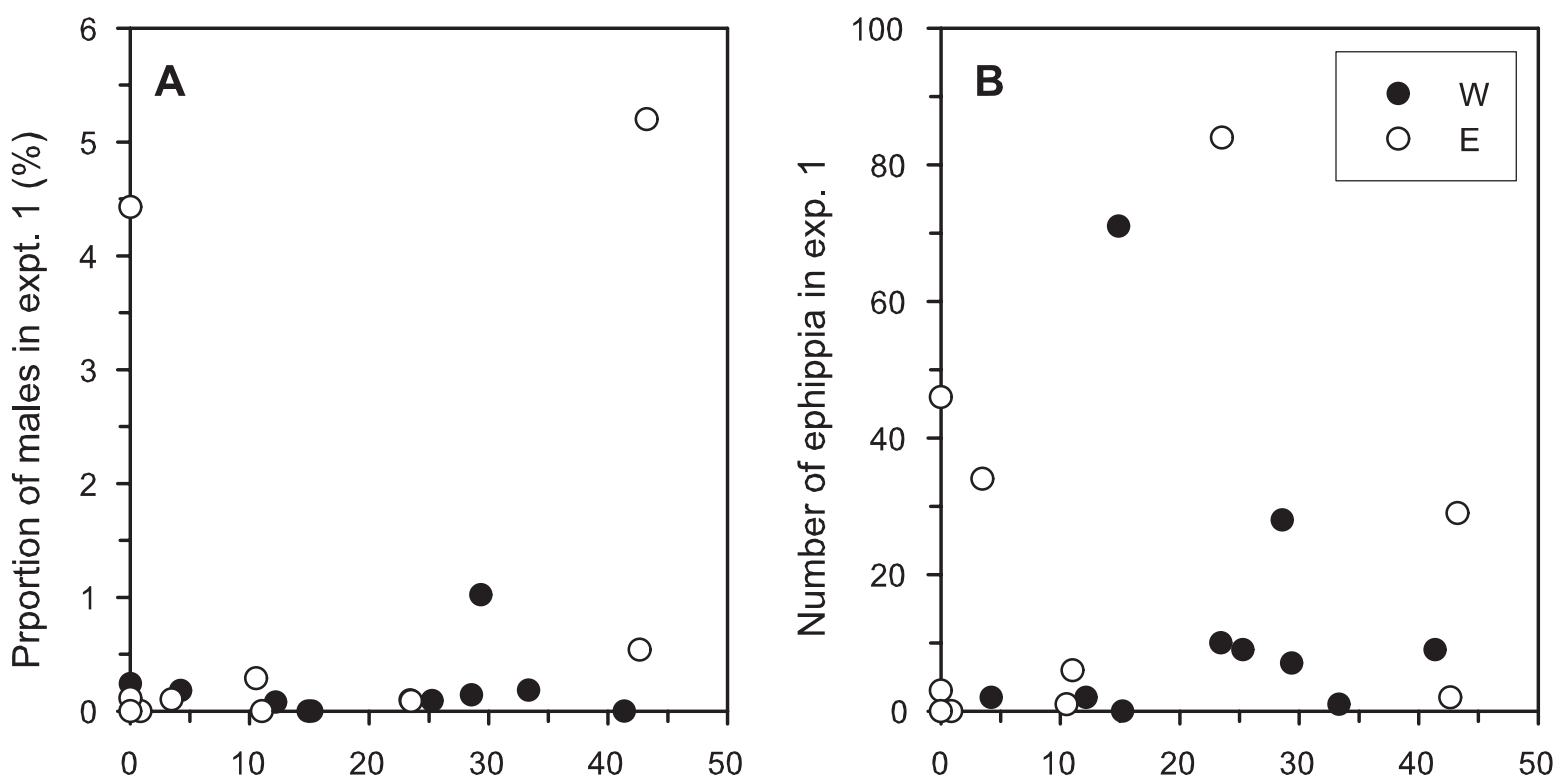

Proportion of males in expt. 2 (\%)

Fig. 6. Relationships between the proportions of male neonates produced in Expt. 2 and the proportions of males found at the termination of Expt. 1 (A) or the total number of ephippia collected in Expt. 1 (B). Each symbol represents a single clone in one of the two groups.

clonal variability (SD) combined with the small sample size. Consequently, the probability of accepting $\mathrm{H}_{0}$ was high, i.e. we could not really reject either of the hypotheses.

\section{Ephippia vs. male production}

Both the proportion of male offspring in Expt. 2 and the number of ephippia produced in Expt. 1 may be considered as traits related to the tendency of sexual reproduction in a particular clone. Therefore, we checked for possible correlations. We found no relationship between the proportion of male offspring in Expt. 2 and the proportion of males at the end of
Expt. 1 (Fig. 6A). Rather the two (E) clones with the highest male abundance in Expt.1 had zero as well as the maximum proportion in Expt. 2. This does not preclude that a relationship may be hidden due to the different sampling. Note that in Expt. 2 we determined the proportion of males among all offspring during 6 days, while the proportion in Expt. 1 refers to the total population at the end of the experiment (cf. Fig. 1). In this case males produced earlier may no longer have been present when the experiment was terminated.

However, the production of males was also not related to the production of ephippia, neither if only the treatment phase of Expt.1 was considered (Fig. 3) nor 
if the total ephippia production in Expt. 1 was compared to the tendency of male production in Expt. 2 (Fig. 6 B). Only one clone in each group produced neither males nor ephippia. The two traits seem to vary independently.

\section{Discussion}

The two experiments used different approaches. Experiment 1 was a population approach, while Expt. 2 was a more physiological approach with better controlled conditions. The latter was necessary as Expt. 1 was more appropriate to measure the tendency of producing ephippia that could be collected throughout the whole experimental period but was not so appropriate to monitor male production, as males could only be collected at the end of the experiment. At this time, males produced in earlier phases may have been dead already.

The basic results were similar in both experiments. We found very large differences between individual clones for both ephippia production and male production. This is consistent with many earlier studies on induction of sexual reproduction in Daphnia as far as they employed a variety of clones (e.g., Hobaek \& Larsson 1990, Larsson 1991, Innes \& Dunbrack 1993, Deng 1996). However, due to the large clonal variability there were no significant differences between the clonal groups. Unfortunately, we cannot rule out that nevertheless differences between groups exist. The low power of the tests does not allow us to make a clear decision for one of the hypotheses. When we designed the experiments, we did not expect such large clonal variability in reproductive parameters. Given the standard deviations of about twice the mean (Table 2), it may require extremely high experimental effort to gain sufficient test power for the reproductive characteristics. An a priori power analysis using the calculated effect sizes predicted necessary sample sizes (number of clones) between 300 (for \% males in Expt. 2) and 1200 (for total ephippia in Expt. 1) in order to gain a power of 0.8 .

On the other hand, differences between means of the two parameters of sexual reproduction did not even show identical trends between groups. There was a tendency to higher ephippia production in E clones but a reverse trend was found for the proportion of males, which was not consistent with our expectations of E clones to exhibit a generally higher tendency towards sexual reproduction. Although the group means of reproductive characteristics seem relatively large, which may be an indication of selection and temporal differentiation we found no evidence for a strong dominance of one of the strategies, parthenogenetic or sexual overwintering, between the egg bank and the open-water population. It is more likely that temporal differentiation is incomplete or individual clones in Myravann use a mixed strategy. This is consistent with the results of genetic analyses and measurements of growth related traits for the two clonal groups (Lampert et al. 2010).

Although we do not have quantitative data, we noticed that many of the ephippia produced in Expt. 1 did not contain dormant eggs, i.e., receptive females had not been fertilized. Large proportions of empty ephippia have been found earlier (Larsson 1991) and they have been taken as evidence for uncoupling of male and ephippia production. Fertilization efficiency in our population experiment was evidently low, although Expt. 1 was designed to include several stimuli, i.e., crowding and photoperiod found to affect both ephippia and male production (Innes \& Dunbrack 1993, Fitzsimmons \& Innes 2006) and food deprivation affecting ephippia formation (Kleiven et al. 1992, La Montagne \& McCauley 2001). As we tested clonal lines this may be the result of a mechanism to prevent inbreeding either by reduced fertilization success or by production of low numbers of males due to nonmatching external stimuli. There is evidence of fitness disadvantage of inbreeding in Daphnia that drives selection against selfing. Laboratory crossing experiments (Innes 1989, Larsson 1991, Innes \& Dunbrack 1993) showed strong inbreeding depression (40\% lowered survival) for selfed offspring of D. obtusa and D. pulex, respectively. In the field, De Meester \& Vanoverbeke (1999) inferred low selfing incidences in D. magna populations from allozyme studies. Ebert et al. (2007) demonstrated the benefit of outcrossing in Daphnia magna gained from higher genetic diversity when confronted with parasites. However, Winsor \& Innes (2002) combining male and female D. pulex of the same or different clones did not find differences in mating proportions, i.e. there was no behavioural inbreeding avoidance. Inbreeding avoidance may rather be achieved by an intraclonal mismatch of the induction of males and sexual eggs (De Meester \& Vanoverbeke 1999). The lack of a correlation between male proportions and ephippia production in our data supports this line of reasoning. The low fertilization success in Expt. 1 may be caused by a lack of males. In fact, proportions of males in Expt. 1 were very low. Only two E clones showed more than $1 \%$ males at the end of the experiment (Fig. 3). 
A more realistic estimate of the potential for male production of the clones was probably detected in Expt. 2 where the proportion of male offspring was measured directly. A single factor, short daylength, was sufficient to induce proportions of up to $40 \%$ male offspring in some clones. These proportions are similar to the values reported by Hobaek \& Larsson (1990) and Larsson (1991) for short-day $(\mathrm{L}: \mathrm{D}=8: 16 \mathrm{~h})$ conditions. Although maximum male proportions were similar in both clonal groups, the differences among clones were more equally distributed in the W group than in the E group (Fig. 5). Males were more often completely missing among E clones. This is not consistent with our expectations that $\mathrm{E}$ clones would show a greater tendency for sexual reproduction. However, although short-day conditions have frequently been shown to induce males, the clone-specific male production may be influenced by additional environmental cues (Larsson 1991, Innes \& Dunbrack 1993). Hence, clones may react differently in the field if crowding or adverse food conditions act in addition to light.

We found no correlation of the two traits of sexual reproduction (ephippia and male production) neither within Expt. 1 (Fig. 3) nor between experiments (Fig. 6). A single male was found for the (E) clone with the highest ephippia production in Expt. 1. Clones that produced many males in Expt. 2 did not show the same pattern at the end of Expt. 1. Already Yampolsky (1992) found no genetic correlation between male frequency and ephippium production in laboratory experiments with $D$. magna. He explained the absence of a negative correlation with the lack of selection in a large population with high clonal diversity as the chance of mating with the same clone is low. In fact, population genetic studies of the $D$. pulex population in Myravann (K. P. Lampert, unpubl.) revealed very high clonal diversity in the field.

When comparing sexual investment for a large number of $D$. pulicaria clones from 9 lake populations under two environments Tessier \& Caceres (2004) found a similar pattern as in our Fig. 6 B: no correlation between male and ephippia production, and various clones that produced either only males or only ephippia. They noted a 'surprisingly large number of clones that were apparently gender specialists'. Tessier \& Caceres (2004) assigned the clones to 4 categories of reproductive investment. Considering that they combined 271 data points compared to only 21 in our Fig. $6 \mathrm{~B}$, the results of the categorization (rounded $\%$ in each category) show a similar trend in the two studies (our data in parentheses): ephippia and males
- 51 (71); only males - 20 (10); only - 11 (10); no sex -18 (10). This is remarkable as Tessier \& Caceres (2004) purposely combined lakes with differing predation risk (defined as 'summer refuge') for the Daphnia summer population, and the variability between lakes was very large. They concluded that the interlake variability originates from sex induction having a genetic basis that is subject to selection according to the overall risk.

On the contrary, we looked at a single population where the risk may vary temporally and sexual investment may depend on the predictability of catastrophic events. At the time when we isolated our clones, predation by planktivorous fish was very low in Myravann. Hence we assumed that ephippia production in this lake was rather an adaptation to critical environmental factors than to fish predation. However, although we found significant genetic variability for traits related to spring success (growth reaction norms; Lampert et al. 2010) as well as for traits of sexual reproduction proof for differences between group means was insufficient. There is still the possibility that a much larger sample size of clones may have revealed subtle differences, but the similarity of our results with those of Tessier \& Caceres (2004) employing many more clones reduces such expectations.

Although clones of Daphnia that reproduce by obligate parthenogenesis and produce resting eggs without fertilization have been described for particular habitats (Hebert \& Crease 1983) and sexual reproduction seems to be less important as overwintering strategy in large, deep lakes (Rellstab \& Spaak 2009), the absence of both male and ephippia production in a small proportion of clones in laboratory experiments (Tessier \& Caceres 2004, this study) is no proof for the existence of clones without any sex competing with cyclic parthenogens in the source lakes. Such permanently parthenogenetic clones would be faced with the risk of extinction from the accumulation of deleterious mutations by Muller's ratchet (Muller 1964). Cyclic parthenogenesis offers the possibility to save a large part of the costs of sexual reproduction, and at the same time purge deleterious mutations (Lynch \& Gabriel 1983). This may be an additional factor preventing Daphnia from skipping the costly production of dormant resting stages and specializing in parthenogenetic overwintering.

Being a small lake, Myravann represents a habitat less predictable than large lakes, but the probability of catastrophic events is still considerably lower than in ponds. Hence, sexual reproduction may play an intermediate role. This assumption is supported by an 
earlier study (Giske 1986) reporting low sexual activity in Myravann. Ephippia were mainly produced in autumn, but of 593 egg-bearing females of $D$. pulex collected in November/December only 11 carried an ephippium.

Using traits directly related to sexual reproduction, the present study supports our conclusions drawn from the genetic structure and from growth related traits (Lampert et al. 2010). We cannot ignore the possibility of temporal differentiation in physiological, reproductive and genetic traits between the two groups of clones. However our results, in comparison with literature data, suggest that D. pulex in Myravann also used a mixed strategy, i.e. individual females receiving the proper cue reproduced sexually and deposited dormant resting stages as 'insurance', and then continued with parthenogenetic reproduction trying to survive the winter and use the advantage of being early in spring. This may be considered a proper strategy if the chance of surviving the winter as a parthenogenetic female under the ice is poorly predictable.

\section{Acknowledgements}

This is the second paper presenting results of a cooperative project between the University of Bergen, Norway, the Max Planck Institute for Evolutionary Biology at Plön, Germany, and the University of Bochum, Germany. The project was only possible through the temporary employment of W. L. as Professor II by the University of Bergen. We are grateful to Knut-Helge Jensen for maintaining the Daphnia clones, for help in the laboratory, valuable suggestions and fruitful discussions. Per Jakobsen and two anonymous referees contributed important ideas, and B. Haubold provided statistical advice. The Melzer Foundation provided funds to prepare this project. The genetic work was funded by a grant of the Deutsche Forschungsgemeinschaft (LA 1382/3-1) to K. P. L.

\section{References}

De Meester, L. \& Vanoverbeke, J., 1999: An uncoupling of male and sexual egg production leads to reduced inbreeding in the cyclical parthenogen Daphnia. - Proc. R. Soc. London Ser. B 266: 2471-2477.

Deng, H.-W., 1996: Environmental and genetic control of sexual reproduction in Daphnia. - Heredity 76: 449-478.

Ebert, D., Altermatt, F. \& Lass, S., 2007: A short term benefit for outcrossing in a Daphnia metapopulation in relation to parasitism. - J. R. Soc. Interface 4: 777-785.

Faul, F., Erdfelder, E., Lang, A.-G., \& Buchner, A. (2007). $\mathrm{G}^{*}$ Power 3: A flexible statistical power analysis program for the social, behavioral, and biomedical sciences. - Behav. Res. Meth. 39: 175-191.

Fitzsimmons, J. M. \& Innes, D. J., 2006: Inter-genotype variation in reproductive response to crowding among Daphnia pulex. - Hydrobiologia 568: 187-205.

Giske, J., 1986: Populasjonsregulerende faktorer hos to arter Daphnia i et vatn uten planktivor fisk. - Master thesis, University of Bergen, Norway.

Haney, J. F. \& Hall, D. J., 1973: Sugar coated Daphnia: A preservation technique for Cladocera. - Limnol. Oceanogr. 18: 331-333.

Hebert, P. D. N. \& Crease, T., 1983: Clonal diversity in populations of Daphnia pulex reproducing by obligate parthenogenesis. - Heredity 51: 353-369.

Hines, J., 2000: NCSS 2000. - NCSS Statistical Software, Kaysville, Utah, USA.

Hobaek, A. \& Larsson, P., 1990: Sex determination in Daphnia magna. - Ecology 71: 2255-226.

Howell, D. C., 2000: Resampling Procedures (v. 1.3). - http:// www.uvm.edu/ dhowell/StatPages/Resampling/Resampling.html (last visited 09.26.2011).

Innes, D. J., 1989: Genetics of Daphnia obtusa: Genetic load and linkage analysis in a cyclical parthenogen. - J. Hered. 80: $6-10$.

Innes, D. J. \& Dunbrack, R. L., 1993: Sex allocation variation in Daphnia pulex. - J. Evol. Biol. 6: 559-575.

Kleiven, O. T., Larsson, P. \& Hobaek, A., 1992: Sexual reproduction in Daphnia magna requires three stimuli. - Oikos 65: 197-206.

Knudsen, F. R., Larsson, P. \& Jakobsen, P. J., 2006. Acoustic scattering from a larval insect (Chaoborus flavicans) at six echosounder frequencies: Implications for acoustic estimates of fish abundance. - Fish. Res. 79: 84-89.

La Montagne, J. M. \& McCauley, E., 2001: Maternal effects in Daphnia: what mothers are telling their offspring and do they listen? - Ecol. Lett. 4: 64-71.

Lampert, W., Lampert, K. P. \& Larsson, P., 2010: Coexisting overwintering strategies in Daphnia pulex: A test of genetic differences and growth responses. - Limnol. Oceanogr. 55: 1893-1900.

Larsson, P., 1991: Intraspecific variability in response to stimuli for male and ephippia formation in Daphnia pulex. - Hydrobiologia 225: 281-290.

Lynch, M. \& Gabriel, W., 1983: Phenotypic evolution and parthenogenesis. - Am. Nat. 122: 745-764.

Muller, H. J., 1964: The relation of recombination to mutational advance. - Mut. Res. 1: 2-9.

Rellstab, C. \& Spaak, P., 2009: Lake origin determines Daphnia population growth under winter conditions. - J. Plankton Res. 31: 261-271.

Tessier, A. J. \& Caceres, C. E., 2004: Differentiation in sex investment by clones and populations of Daphnia. - Ecol. Lett. 7: 695-703.

Winsor, G. L. \& Innes, D. J., 2002: Sexual reproduction in Daphnia pulex (Crustacea: Cladocera): observations on male mating behaviour and avoidance of inbreeding. - Freshwat. Biol. 47: 441-450.

Yampolsky, L. Y., 1992: Genetic variation in the sexual reproduction rate within a population of a cyclic parthenogen, Daphnia magna. - Evolution 46: 833-837.

Zaffagnini, F., 1987: Reproduction in Daphnia. - Mem. Ist. ital. Idrobiol. 45: 245-284.

Submitted: 30 June 2011; accepted: 19 December 2011. 